\title{
COVID-19 and Liver Injury: A Systematic Review and Meta-Analysis
}

Jawad Ahmed ${ }^{1}$, Tehlil Rizwan ${ }^{1}$, Farheen Malik ${ }^{1}$, Raniyah Akhter ${ }^{2}$, Mehreen Malik ${ }^{3}$, Junaid Ahmad ${ }^{4}$, Abdul Wasay Khan ${ }^{5}$, Muhammad A. Chaudhary 6, 7 , Muhammad Shariq Usman ${ }^{8}$

1. Internal Medicine, Dow University of Health Sciences, Karachi, PAK 2. Pulmonology, Fazaia Ruth Phau Medical College, Pakistan Air Force (PAF) Hospital, Karachi, PAK 3. Anesthesiology, Aga Khan University, Karachi, PAK 4. Internal Medicine, Liaquat University of Medical and Health Sciences, Jamshoro, PAK 5. Pediatrics, University of Kansas School of Medicine - Wichita, Wichita, USA 6. Family Medicine, WellSpan Good Samaritan Hospital, Lebanon, USA 7. Center for Surgery and Public Health, Harvard Medical School/Harvard T. H. Chan School of Public Health, Boston, USA 8. Internal Medicine, Civil Hospital Karachi, Dow University of Health Sciences, Karachi, PAK

Corresponding author: Jawad Ahmed, jawadahmedd13@gmail.com

\section{Abstract}

\section{Background and Aims}

The prevalence and extent of liver damage in coronavirus disease 2019 (COVID-19) patients remain poorly understood, primarily due to small-sized epidemiological studies with varying definitions of "liver injury". We conducted a meta-analysis to derive generalizable, well-powered estimates of liver injury prevalence in COVID-19 patients. We also aimed to assess whether liver injury prevalence is significantly greater than the baseline prevalence of chronic liver disease (CLD). Our secondary aim was to study whether the degree of liver injury was associated with the severity of COVID-19.

\section{Materials and Methods}

Electronic databases (PubMed and Scopus) were systematically searched in June 2020 for studies reporting the prevalence of baseline CLD and current liver injury in hospitalized COVID-19 patients. Liver injury was defined as an elevation in transaminases $>3$ times above the upper limit of normal. For the secondary analysis, all studies reporting mean liver enzyme levels in severe versus non-severe COVID-19 patients were included. A random-effects model was used for meta-analysis. Proportions were subjected to arcsine transformation and pooled to derive pooled proportions and corresponding 95\% confidence intervals (CIs). Subgroup differences were tested for using the chi-square test and associated p-value. Means and their standard errors were pooled to derive weighted mean differences (WMDs) and corresponding 95\% CIs.

\section{Results}

Electronic search yielded a total of 521 articles. After removal of duplicates and reviewing the full-texts of potential studies, a total of 27 studies met the inclusion criteria. Among a cohort of 8,817 patients, the prevalence of current liver injury was $15.7 \%$ (9.5\%-23.0\%), and this was significantly higher than the proportion of patients with a history of CLD $(4.9 \%$ [2.2\%-8.6\%]; $\mathrm{p}<0.001)$. A total of 2,900 patients in our

Received 07/13/2020 Review began 07/16/2020 Review ended 07/16/2020 Published 07/27/2020

\section{() Copyright 2020}

Ahmed et al. This is an open access article distributed under the terms of the Creative Commons Attribution License CC-BY 4.0., which permits unrestricted use, distribution, and reproduction in any medium, provided the original author and source are credited. population had severe COVID-19, and 7,184 patients had non-severe COVID-19. Serum ALT (WMD: 7.19 [4.90, 9.48]; $\mathrm{p}<0.001 ; \mathrm{I}^{2}=69 \%$ ), AST (WMD: 9.02 [6.89, 11.15]; $\mathrm{p}<0.001 ; \mathrm{I}^{2}=73 \%$ ) and bilirubin levels (WMD: $1.78[0.86,2.70] ; \mathrm{p}<0.001 ; \mathrm{I}^{2}=82 \%$ ) were significantly higher in patients with severe COVID-19 when compared to patients with non-severe disease. Albumin levels were significantly lower in patients with severe COVID-19 (WMD: -4.16 [-5.97, -2.35]; $\left.\mathrm{p}<0.001 ; \mathrm{I}^{2}=95 \%\right)$.

\section{Conclusions}

Patients with COVID-19 have a higher than expected prevalence of liver injury, and the extent of the injury is associated with the severity of the disease. Further studies are required to assess whether hepatic damage is caused by the virus, medications, or both.

Categories: Internal Medicine, Gastroenterology, Infectious Disease

Keywords: liver injury, liver abnormalities, coronavirus disease 2019, covid-19, liver injury biomarkers, liver enzymes, hepatic injury, sars-cov-2

\section{Introduction}

Severe acute respiratory syndrome coronavirus 2 (SARS-Cov-2), the virus responsible for coronavirus disease 2019 (COVID-19), mainly affects the respiratory system causing symptoms of fever, fatigue, cough, dyspnea, loss of appetite, muscle and joint pains [1]. However, the incidence of vomiting, nausea, and diarrhea have also been reported, suggesting the involvement of gastrointestinal and hepatobiliary systems [1,2]. SARSCov-2 enters cells through the angiotensin-converting enzyme-2 (ACE2) protein. Apart from type II alveolar 
epithelial cells of the lung, ACE2 protein is also expressed in the bile ducts cells [3]. This suggests that SARS-Cov-2 could potentially infect bile duct cells and cause abnormal liver function tests. According to one recent study, liver biopsy specimens of COVID-19 patients demonstrated moderate microvascular steatosis and mild lobular and portal activity, suggesting liver injury [4].

Although, in theory, liver injury in COVID-19 patients is possible, the actual prevalence and extent of liver damage in these patients remain poorly understood. This is primarily because most published COVID-19 studies are small-sized, often lack adjustment for baseline chronic liver disease (CLD), and have inconsistent definitions of "liver injury". In this meta-analysis, we aim to provide well-powered and generalizable estimates of the prevalence of liver injury in COVID-19 patients while making sure to keep the definition of "liver injury" consistent. We also seek to assess whether the prevalence of liver injury in COVID-19 patients is significantly different from the prevalence of baseline CLD in these patients. A secondary aim of this study is to assess for any significant differences in serum biomarkers of liver injury (alanine transaminase [ALT], aspartate transaminase [AST], total bilirubin, and albumin) in patients with severe versus non-severe COVID-19.

\section{Materials And Methods}

This meta-analysis was conducted according to the Preferred Reporting Items for Systemic Reviews and Meta-Analyses (PRISMA) guidelines [5].

\section{Literature search}

PubMed and Scopus were searched from the inception of databases till June 18, 2020, using the following search string: ("novel coronavirus" OR "2019-nCoV" OR "severe acute respiratory syndrome coronavirus 2" OR “SARS-CoV-2” OR “coronavirus disease 2019” OR “COVID-19”) AND (“Aspartate Aminotransferases” OR "SGOT” OR "Alanine Transaminase" OR "Alanine aminotransferase” OR "SGPT” OR "Albumin” OR "Bilirubin” OR “Liver”) AND ("hepatic injury” OR "liver injury” OR "liver damage” OR "liver abnormality"). Google Scholar was also searched for grey literature. No language and time restrictions were set. The search strategy for both databases is shown in Table 1 .

\section{Electronic database \\ Search strategy}

(((()("novel coronavirus"[All Fields] OR "2019-nCoV"[All Fields]) OR "severe acute respiratory syndrome coronavirus 2"[All Fields]) OR "SARS-CoV-2" [All Fields]) OR "coronavirus disease 2019"[All Fields]) OR "COVID-19"[All Fields]) AND (()(((")Aspartate Aminotransferases"[All Fields] OR "SGOT"[All Fields]) OR "Alanine Transaminase"[All Fields]) OR "Alanine aminotransferase"[All Fields]) OR "SGPT"[All Fields]) OR "Albumin"[All Fields]) OR "Bilirubin"[All Fields]) OR "Liver"[All Fields])) AND ((("hepatic injury"[All Fields] OR "liver injury"[All Fields]) OR "liver damage"[All Fields]) OR "liver abnormality"[All Fields])

( TITLE-ABS-KEY ( "liver injury" OR " liver failure" OR "hepatic damage" OR "liver function abnormality" OR "hepatic abnormality") AND TITLE-ABS-KEY ( "ALT" OR " alanine transaminase" OR "SGPT" ) AND TITLE-ABS-KEY ( "AST"

Scopus OR " Aspartate transaminase" OR "SGOT") AND TITLE-ABS-KEY ("bilirubin" OR " total bilirubin") AND TITLE-ABSKEY ( "albumin" OR "serum albumin") AND TITLE-ABS-KEY ("COVID-19" OR "SARS-CoV-2" OR "coronavirus disease" ))

\section{TABLE 1: Search strategy for electronic databases}

\section{Study selection}

All the articles were exported to EndNote Reference Library version X4 (Clarivate Analytics, Philadelphia, PA) for screening and removal of duplicates. Studies were narrowed down based on titles and abstracts, and final inclusion was performed after reviewing the full texts of articles. Studies were selected independently by two reviewers, and a third reviewer resolved any conflict regarding inclusion.

\section{Inclusion criteria and definitions}

We included all studies among COVID-19 patients that defined liver injury as an elevation in transaminases $>3$ times above the upper limit of normal (ULN). To study whether the extent of liver injury was associated with severity of COVID-19, all studies that reported serum ALT, AST, total bilirubin, or albumin levels in severe versus non-severe COVID-19 patients were included. Most of the studies assessed COVID-19 severity according to either the World Health Organization interim guidance for COVID-19 or the guidelines for the diagnosis and management of COVID-19 by the National Health Commission of China, thus ensuring minimum heterogeneity in severity criteria. Case reports and studies that did not report the definition of liver injury were excluded. 


\section{Data extraction}

Data extraction was performed independently by two reviewers, and, in case of any conflict, the opinion of a third reviewer was sought. Characteristics of included studies, patients' baseline information, liver injury biomarkers, and criteria for liver injury were extracted from the studies on a predesigned form.

\section{Statistical analysis}

Open MetaAnalyst and Review Manager Version 5.4 were used for all statistical analyses. Proportions from studies were subjected to arcsine transformation and pooled using a random-effects model to derive the pooled proportions and corresponding $95 \%$ confidence intervals (CIs). The chi-square test was used to assess for any significant differences between baseline CLD prevalence and prevalence of liver injury. Continuous variables were also pooled using a random-effects model to derive the weighted mean difference (WMD) and 95\% CIs. The Higgins I2 statistic was used to evaluate heterogeneity and a value of $25 \%-50 \%$ was considered mild, 50\%-75\% as moderate, and $>75 \%$ as severe heterogeneity. Publication bias was assessed through visual inspection of the funnel plot. A p-value of less than 0.05 was considered significant in all cases.

\section{Results}

The initial search yielded 513 potential articles, and eight records were identified through references of relevant studies. After exclusions, 27 studies were used in our quantitative analysis [1,2,4,6-29]. The PRISMA flowchart (Figure 1) summaries the results of our literature search. The baseline characteristics and outcomes of included studies are given in Table 2. Visual inspection of the funnel plot (based on serum ALT outcome) showed no publication bias (Figure 2).

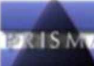

\section{PRISMA 2009 Flow Diagram}
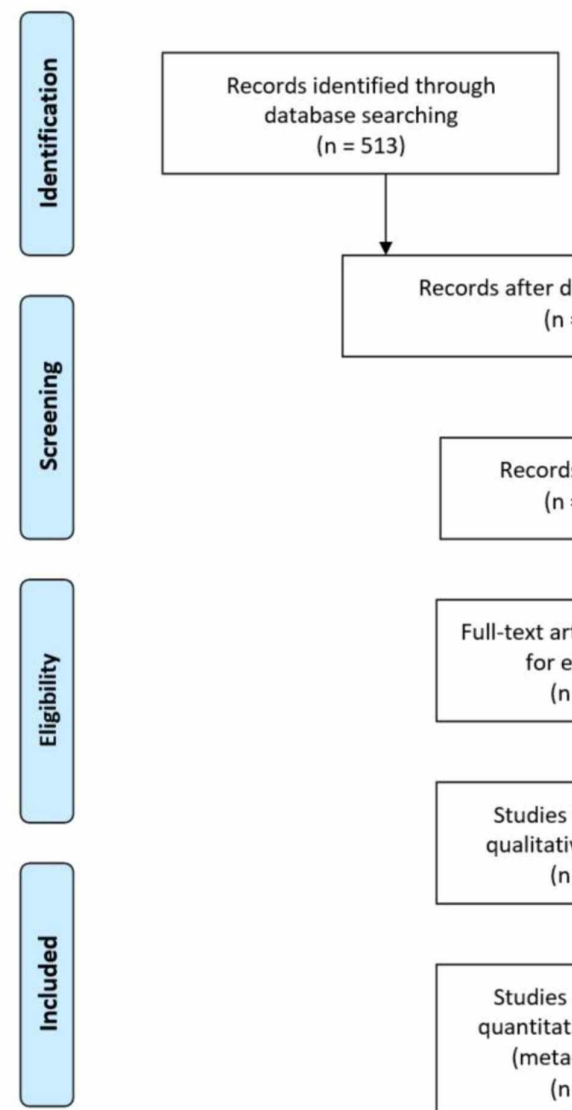
atabase searchin

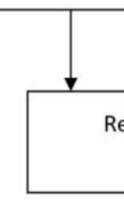

Records after duplicates removed

Additional records identified through other sources $(n=8)$

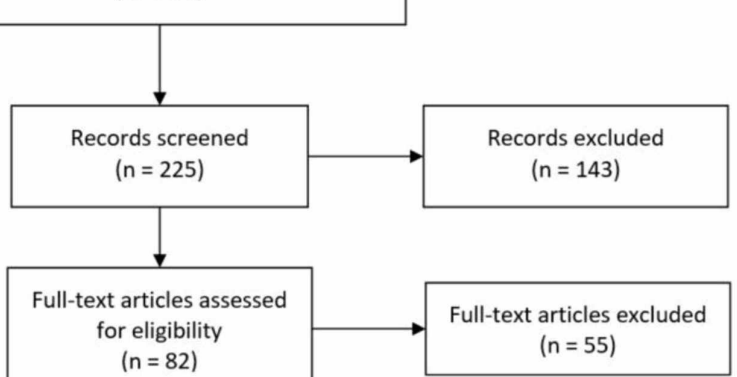

FIGURE 1: PRISMA flow chart summarizing the literature search

PRISMA, Preferred Reporting Items for Systemic Reviews and Meta-Analyses 


\begin{tabular}{|c|c|c|c|c|c|c|c|}
\hline Author name & Location & Study design & Total & $\begin{array}{l}\text { Males } \\
(\%)\end{array}$ & $\begin{array}{l}\text { Age } \\
\text { (years) }\end{array}$ & $\begin{array}{l}\text { Baseline CLD } \\
(\%)\end{array}$ & Outcomes \\
\hline $\begin{array}{l}\text { Bloom et } \\
\text { al. [2] }\end{array}$ & USA & Prospective & 60 & $39(65.0)$ & 57 & $4(7.0)$ & Liver injury \\
\hline Cai et al. [6] & China & Retrospective & 298 & $\begin{array}{l}145 \\
(48.7)\end{array}$ & 47.5 & $28(9.4)$ & $\begin{array}{l}\text { Liver injury; biomarkers: ALT, AST, and T. } \\
\text { bilirubin }\end{array}$ \\
\hline Cai et al. [7] & Chına & $\begin{array}{l}\text { Cross- } \\
\text { sectional }\end{array}$ & 417 & $\begin{array}{l}298 \\
(71.5)\end{array}$ & 47.32 & $21(5.04)$ & $\begin{array}{l}\text { Liver injury; biomarkers: ALT, AST, and T. } \\
\text { bilirubin }\end{array}$ \\
\hline Chen et al. [8] & China & Retrospective & 21 & $17(81.0)$ & 56.5 & NR & $\begin{array}{l}\text { Biomarkers: ALT, AST, T. bilirubin, and } \\
\text { albumin }\end{array}$ \\
\hline Chen et al. [4] & China & Retrospective & 274 & $\begin{array}{l}171 \\
(62.0)\end{array}$ & 59.5 & NR & $\begin{array}{l}\text { Biomarkers: ALT, AST, T. bilirubin, and } \\
\text { albumin }\end{array}$ \\
\hline Deng et al. [9] & China & Retrospective & 225 & $\begin{array}{l}124 \\
(55.1)\end{array}$ & 54.5 & NR & Biomarkers: ALT and AST \\
\hline Fu et al. [10] & China & Retrospective & 355 & $\begin{array}{l}190 \\
(53.5)\end{array}$ & $>60=115$ & $9(2.5)$ & $\begin{array}{l}\text { Biomarkers: ALT, AST, T. bilirubin, and } \\
\text { albumin }\end{array}$ \\
\hline Gao et al. [11] & China & Retrospective & 43 & $26(60.5)$ & 44.08 & NR & Biomarkers: ALT and AST \\
\hline $\begin{array}{l}\text { Huang C et } \\
\text { al. [1] }\end{array}$ & China & Retrospective & 41 & $30(73.0)$ & 49 & $1(3.6)$ & Biomarkers: ALT and AST \\
\hline Jin et al. [12] & China & Retrospective & 651 & $\begin{array}{l}331 \\
(50.8)\end{array}$ & 45.61 & $25(3.8)$ & $\begin{array}{l}\text { Biomarkers: ALT, AST, T. bilirubin, and } \\
\text { albumin }\end{array}$ \\
\hline Lei et al. [13] & China & Retrospective & 5,771 & $\begin{array}{l}2,724 \\
(47.2)\end{array}$ & 56 & $81(1.4)$ & Liver injury \\
\hline Liu et al. [14] & China & Retrospective & 78 & $39(50.0)$ & 51.5 & NR & Biomarkers: ALT, AST, and albumin \\
\hline Mo et al. [15] & China & Retrospective & 155 & $86(55.5)$ & 53.5 & $7(4.5)$ & Biomarkers: ALT, AST, and albumin \\
\hline Pan et al. [16] & China & Retrospective & 204 & $\begin{array}{l}107 \\
(52.5)\end{array}$ & 52.9 & $2(0.01)$ & $\begin{array}{l}\text { Biomarkers: ALT, AST, T. bilirubin, and } \\
\text { albumin }\end{array}$ \\
\hline $\begin{array}{l}\text { Phipps et } \\
\text { al. [17] }\end{array}$ & USA & Retrospective & 2,273 & $\begin{array}{l}1,297 \\
(57.1)\end{array}$ & 65 & $114(5.0)$ & Liver injury \\
\hline Qian et al. [18] & China & Retrospective & 91 & $37(40.7)$ & 57.5 & NR & Biomarkers: ALT, AST, and albumin \\
\hline Qu et al. [19] & China & Retrospective & 30 & $16(53.3)$ & 54.7 & Excluded (0) & Biomarkers: ALT and AST \\
\hline $\begin{array}{l}\text { Ruan et } \\
\text { al. [20] }\end{array}$ & China & Retrospective & 150 & $102(68)$ & 58.5 & $4(2.7)$ & Biomarkers: T. bilirubin and albumin \\
\hline Wan et al. [21] & China & Retrospective & 135 & 72 (53.3) & 50 & $2(1.5)$ & $\begin{array}{l}\text { Biomarkers: ALT, AST, T. bilirubin, and } \\
\text { albumin }\end{array}$ \\
\hline $\begin{array}{l}\text { Wang et } \\
\text { al. [22] }\end{array}$ & China & Retrospective & 138 & 75 (54.3) & 58.5 & $4(2.9)$ & Biomarkers: ALT, AST, and T. bilirubin \\
\hline Wang et & China & Retrospective & 69 & 32 (46) & 53.7 & $1(1.4)$ & Biomarkers: ALT and AST \\
\hline Wu et al. [24] & China & Retrospective & 201 & $\begin{array}{l}128 \\
(63.7)\end{array}$ & 53.25 & $7(3.5)$ & $\begin{array}{l}\text { Biomarkers: ALT, AST, T. bilirubin, and } \\
\text { albumin }\end{array}$ \\
\hline Xie et al. [25] & China & Retrospective & 79 & $44(55.7)$ & 60 & Excluded (0) & Biomarkers: ALT, AST, and T. bilirubin \\
\hline $\begin{array}{l}\text { Yang et } \\
\text { al. [26] }\end{array}$ & China & Retrospective & 52 & $35(67.0)$ & 58.25 & $16(30.8)$ & Biomarkers: T. bilirubin \\
\hline $\begin{array}{l}\text { Zhang et } \\
\text { al. [27] }\end{array}$ & China & Retrospective & 645 & $\begin{array}{l}328 \\
(50.9)\end{array}$ & 40.77 & $25(3.9)$ & $\begin{array}{l}\text { Biomarkers: ALT, AST, T. bilirubin, and } \\
\text { albumin }\end{array}$ \\
\hline $\begin{array}{l}\text { Zhou et } \\
\text { al. [28] }\end{array}$ & China & Retrospective & 34 & $17(50.0)$ & 65 & NR & and AS \\
\hline
\end{tabular}




\section{Cureus}
Zhou et
China
Retrospective 191
119
(62.0)
60.5
NR
Biomarkers: ALT and albumin

\section{TABLE 2: Baseline characteristics and demographics of the included studies}

CLD, chronic liver disease; ALT, alanine transaminase; AST, aspartate transaminase; T. bilirubin, total bilirubin; NR, not reported

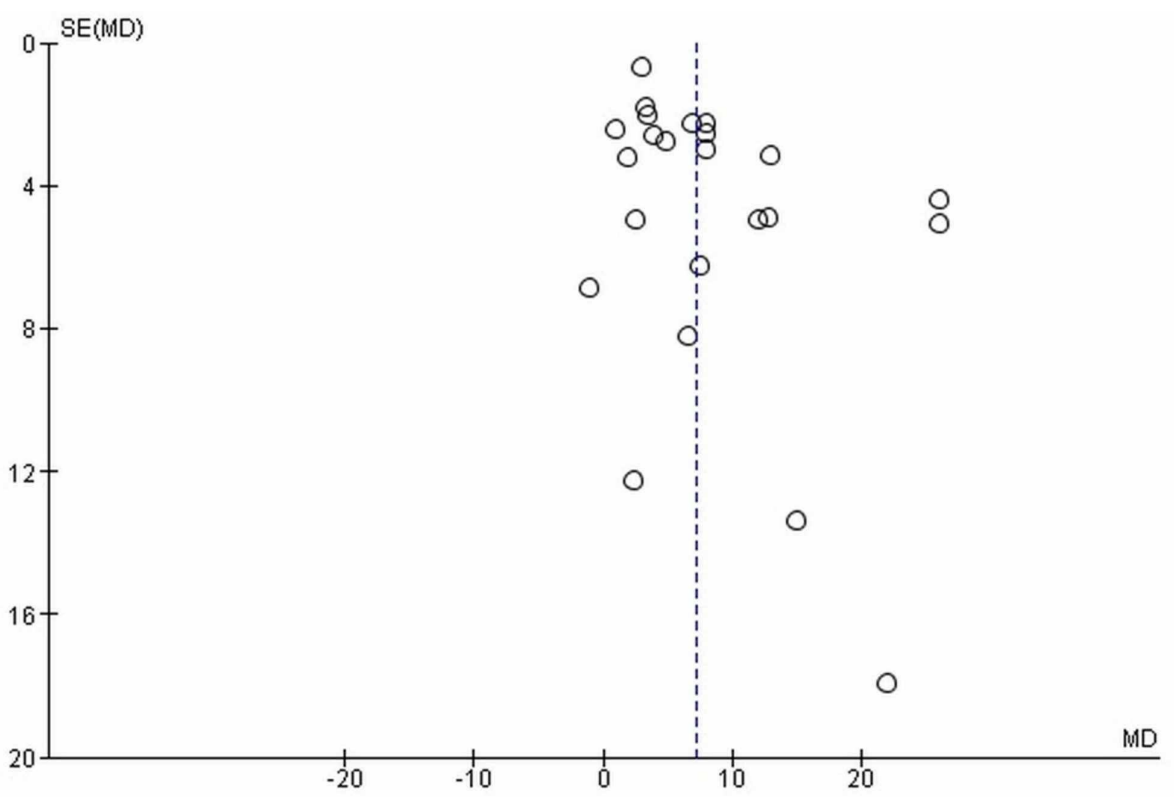

\section{FIGURE 2: Funnel plot for publication bias}

The funnel plot is based on serum ALT levels.

SE, standard error; MD, mean difference; ALT, alanine transaminase

\section{Liver injury}

Liver injury was defined as an elevation in transaminases $>3$ times above the ULN. Five studies, including 8,817 COVID-19 positive patients, met the pre-defined inclusion criteria for liver injury $[2,6,7,13,17]$. The pooled proportion of patients with a history of CLD was $4.9 \%(2.2 \%-8.6 \%])$ The prevalence of liver injury was $15.7 \%$ (9.5\%-23.0\%), as shown in Figure 3, and this was significantly higher than the baseline CLD prevalence $(\mathrm{p}<0.001)$. 


\section{Cureus}

A

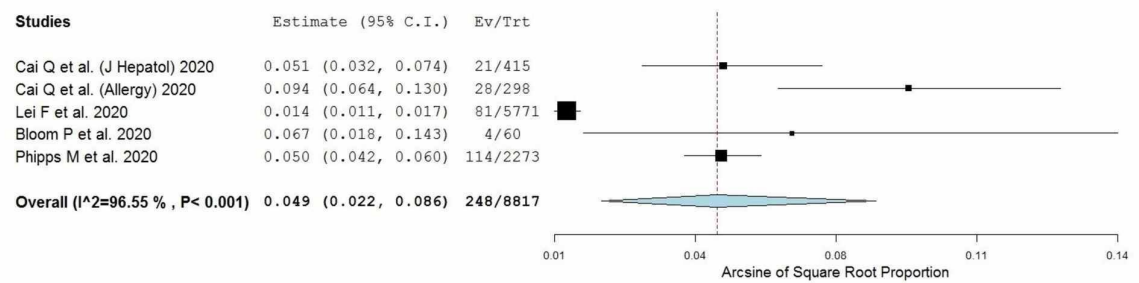

B

Studies

Cai Q et al. (J Hepatol) 2020 Cai $Q$ et al. (Allergy) 2020

Lei $F$ et al. 2020

Bloom P et al. 2020

Phipps M et al. 2020

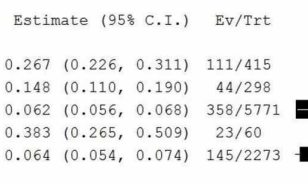

Overall ( $(12=97.89 \%, P<0.001) \quad 0.157 \quad(0.095,0.230) \quad 681 / 8817$

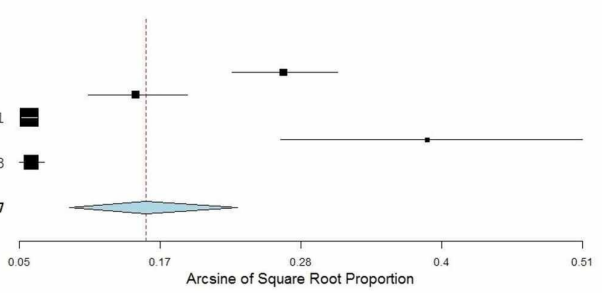

FIGURE 3: Pooled prevalence of (A) patients with a history of chronic liver disease and (B) patients with liver injury

$\mathrm{Cl}$, confidence interval; Ev/Trt, events/total; $\mathrm{I}^{2}$, heterogeneity

\section{Biomarkers of liver injury in severe versus non-severe COVID-19 patients}

Levels of liver biomarkers were reported by 25 studies comprising 10,084 patients (2,900 severe COVID-19 and 7,184 non-severe COVID-19 patients) [1,4,6-16,18-29]. Our analysis shows that serum ALT (WMD: 7.19 [4.90, 9.48]; $\mathrm{p}<0.001 ; \mathrm{I}^{2}=69 \%$ ), AST (WMD: 9.02 [6.89, 11.15]; $\mathrm{p}<0.001 ; \mathrm{I}^{2}=73 \%$ ), and total bilirubin levels (WMD: 1.78 [0.86, 2.70]; $\mathrm{p}<0.001 ; \mathrm{I}^{2}=82 \%$ ) were significantly higher in patients with severe COVID-19 when compared to patients with non-severe disease. Albumin levels were significantly lower in patients with severe COVID-19 (WMD: -4.16 [-5.97, -2.35]; $\mathrm{p}<0.001 ; \mathrm{I}^{2}=95 \%$ ). The individual forest plots for ALT, AST, total bilirubin, and albumin are shown in Figures 4-7, respectively.

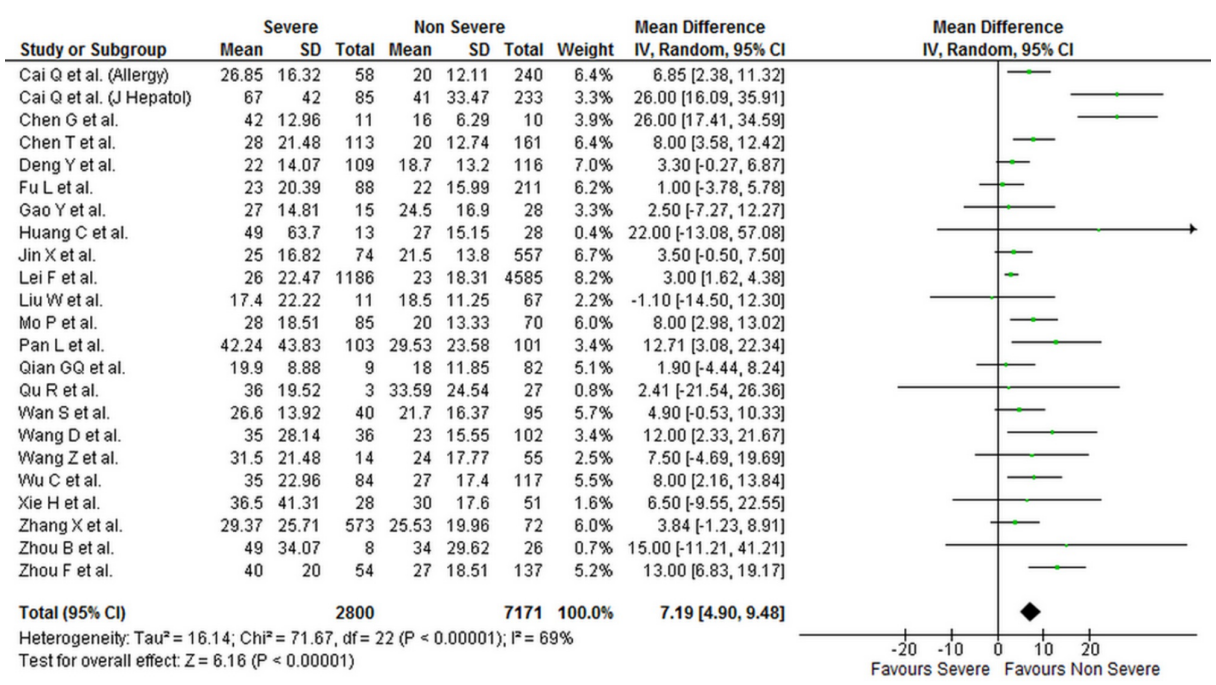

FIGURE 4: Forest plot showing the association between serum ALT levels and severity of disease in COVID-19 patients

SD, standard deviation; IV, inverse variance; Cl, confidence interval; ALT, alanine transaminase; COVID-19, coronavirus disease 2019 


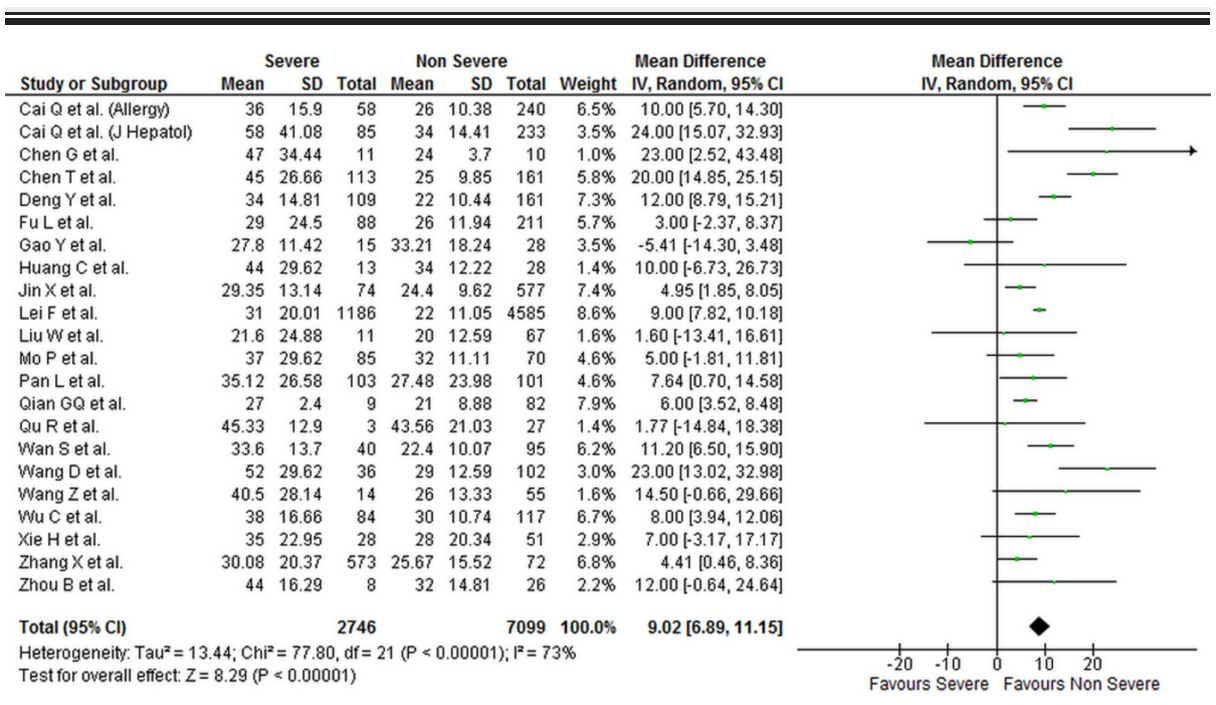

FIGURE 5: Forest plot showing the association between serum AST levels and severity of disease in COVID-19 patients

SD, standard deviation; IV, inverse variance; Cl, confidence interval; AST, aspartate transaminase; COVID-19, coronavirus disease 2019

\begin{tabular}{|c|c|c|c|c|c|c|c|c|c|c|c|}
\hline \multirow[b]{2}{*}{ Study or Subgroup } & \multicolumn{3}{|c|}{ Severe } & \multicolumn{3}{|c|}{ Non Severe } & \multirow[b]{2}{*}{ Weight } & \multirow{2}{*}{$\begin{array}{l}\text { Mean Difference } \\
\text { IV, Random, } 95 \% \mathrm{Cl}\end{array}$} & & \multirow{2}{*}{$\begin{array}{l}\text { Mean Difference } \\
\text { IV, Random, } 95 \% \mathrm{CI}\end{array}$} & \\
\hline & Mean & SD & Total & Mean & SD & Total & & & & & \\
\hline Cai $Q$ et al. (Allergy) & 11.25 & 7.76 & 58 & 10.29 & 1.18 & 240 & $6.5 \%$ & $0.96[-1.04,2.96]$ & & & \\
\hline Cai $Q$ et al. (J Hepatol) & 22 & 7.88 & 85 & 19 & 10.38 & 233 & $6.3 \%$ & $3.00[0.86,5.14]$ & & & \\
\hline Chen $G$ et al. & 8.8 & 1.92 & 11 & 7.8 & 2.29 & 10 & $6.9 \%$ & $1.00[-0.82,2.82]$ & & & \\
\hline Chen T et al. & 12.6 & 4.4 & 113 & 8.4 & 4 & 161 & $8.5 \%$ & $4.20[3.18,5.22]$ & & & \\
\hline Fu Let al. & 10.9 & 6.47 & 88 & 10.4 & 5.75 & 211 & $7.5 \%$ & $0.50[-1.06,2.06]$ & & - & \\
\hline Huang $C$ et al. & 14 & 15.55 & 13 & 10.8 & 2.14 & 28 & $1.0 \%$ & $3.20[-5.29,11.69]$ & & & \\
\hline Jin $\times$ et al. & 10 & 4.92 & 74 & 9.6 & 4.51 & 577 & $8.2 \%$ & $0.40[-0.78,1.58]$ & & - & \\
\hline Lei $F$ et al. & 10.6 & 5.62 & 1186 & 10.3 & 4.84 & 4585 & $9.4 \%$ & $0.30[-0.05,0.65]$ & & $t$ & \\
\hline Pan L et al. & 13.83 & 12.03 & 103 & 13.46 & 8.11 & 101 & $5.0 \%$ & $0.37[-2.44,3.18]$ & & & \\
\hline Ruan $Q$ et al. & 18.1 & 10.7 & 68 & 12.8 & 6.8 & 82 & $4.8 \%$ & $5.30[2.36,8.24]$ & & & \\
\hline Wan $S$ et al. & 9.8 & 5.77 & 40 & 8.6 & 6.22 & 95 & $6.2 \%$ & $1.20[-0.98,3.38]$ & & & \\
\hline Wang $D$ et al. & 11.5 & 6.66 & 36 & 9.3 & 3.4 & 102 & $6.0 \%$ & $2.20[-0.07,4.47]$ & & & \\
\hline Wu C et al. & 12.9 & 5.59 & 84 & 10.5 & 3.74 & 117 & $7.8 \%$ & $2.40[1.03,3.77]$ & & - & \\
\hline Xie $\mathrm{H}$ et al. & 12.7 & 5.6 & 28 & 13.9 & 7.64 & 51 & $4.8 \%$ & $-1.20[-4.15,1.75]$ & & - & \\
\hline Yang $X$ et al. & 19.5 & 11.6 & 32 & 13.1 & 4.3 & 20 & $2.9 \%$ & $6.40[1.96,10.84]$ & & & \\
\hline Zhang $X$ et al. & 11.26 & 8.04 & 573 & 9.11 & 4.86 & 72 & $8.0 \%$ & $2.15[0.85,3.45]$ & & $\longrightarrow$ & \\
\hline Total $(95 \% \mathrm{Cl})$ & & & 2592 & & & 6685 & $100.0 \%$ & $1.78[0.86,2.70]$ & & & \\
\hline \multicolumn{9}{|c|}{$\begin{array}{l}\text { Heterogeneity: } \text { Taú }^{2}=2.30 ; \mathrm{Chi}^{2}=82.45, \mathrm{df}=15(\mathrm{P}<0.00001) ; I^{2}=82 \% \\
\text { Test for overall effect: } Z=3.81(P=0.0001)\end{array}$} & & $\begin{array}{l}-5 \\
\text { ours Severe Favours }\end{array}$ & $\begin{array}{ll}1 & 1 \\
5 & 10 \\
\text { s Non Severe }\end{array}$ \\
\hline
\end{tabular}

FIGURE 6: Forest plot showing the association between serum total bilirubin levels and severity of disease in COVID-19 patients

SD, standard deviation; IV, inverse variance; Cl, confidence interval; COVID-19, coronavirus disease 2019

\begin{tabular}{|c|c|c|c|c|c|c|c|c|c|c|}
\hline \multirow[b]{2}{*}{ Study or Subgroup } & \multicolumn{3}{|c|}{ Severe } & \multicolumn{3}{|c|}{ Non Severe } & \multirow[b]{2}{*}{ Weight } & \multirow{2}{*}{$\begin{array}{l}\text { Mean Difference } \\
\text { IV, Random, } 95 \% \mathrm{Cl}\end{array}$} & \multirow{2}{*}{\multicolumn{2}{|c|}{$\begin{array}{l}\text { Mean Difference } \\
\text { IV, Random, } 95 \% \mathrm{Cl}\end{array}$}} \\
\hline & Mean & SD & Total & Mean & SD & Total & & & & \\
\hline Chen $G$ et al. & 29.6 & 3.25 & 11 & 37.2 & 2.22 & 10 & $7.2 \%$ & $-7.60[-9.96,-5.24]$ & & \\
\hline Chen T et al. & 30.1 & 3.77 & 113 & 36.3 & 4.29 & 161 & $8.1 \%$ & $-6.20[-7.16,-5.24]$ & - & \\
\hline Fu Let al. & 37.3 & 5 & 88 & 40.5 & 5.75 & 211 & $7.9 \%$ & $-3.20[-4.50,-1.90]$ & & \\
\hline Jin $X$ et al. & 40.13 & 4.92 & 74 & 41.5 & 3.8 & 577 & $8.0 \%$ & $-1.37[-2.53,-0.21]$ & & \\
\hline Liu Wet al. & 36.62 & 6.6 & 11 & 41.27 & 4.55 & 67 & $5.8 \%$ & $-4.65[-8.70,-0.60]$ & & \\
\hline Mo $P$ et al. & 36 & 5.92 & 85 & 39 & 4.44 & 70 & $7.7 \%$ & $-3.00[-4.63,-1.37]$ & & \\
\hline Pan L et al. & 36.16 & 6.49 & 103 & 35.84 & 5.63 & 101 & $7.7 \%$ & $0.32[-1.35,1.99]$ & & \\
\hline Qian $G Q$ et al. & 38.55 & 2.16 & 9 & 40.2 & 3.25 & 82 & $7.8 \%$ & $-1.65[-3.23,-0.07]$ & & \\
\hline Ruan Q et al. & 28.8 & 3.8 & 68 & 32.7 & 3.8 & 82 & $8.0 \%$ & $-3.90[-5.12,-2.68]$ & & \\
\hline Wan $S$ et al. & 36 & 4 & 40 & 49.9 & 4.59 & 95 & $7.8 \%$ & $-13.90[-15.45,-12.35]$ & & \\
\hline Wu C et al. & 30.4 & 4.59 & 84 & 33.7 & 3.96 & 117 & $8.0 \%$ & $-3.30[-4.52,-2.08]$ & - & \\
\hline Zhang $X$ et al. & 41.02 & 4.47 & 573 & 42.53 & 4.7 & 72 & $8.0 \%$ & $-1.51[-2.66,-0.36]$ & & \\
\hline Zhou $F$ et al. & 29.1 & 3.55 & 54 & 33.6 & 4.29 & 137 & $8.0 \%$ & $-4.50[-5.69,-3.31]$ & & \\
\hline Total $(95 \% \mathrm{Cl})$ & & & 1313 & & & 1782 & $100.0 \%$ & $-4.16[-5.97,-2.35]$ & & \\
\hline \multicolumn{9}{|c|}{$\begin{array}{l}\text { Heterogeneity: } \mathrm{Tau}^{2}=10.31 ; \mathrm{Chi}^{2}=263.81, \mathrm{df}=12(\mathrm{P}<0.00001) ; \mathrm{I}^{2}=95 \% \\
\text { Test for overall effect: } Z=4.50(P<0.00001)\end{array}$} & $\begin{array}{cc}-1 & 1 \\
-10 & -5 \\
\text { Favours Non Severe }\end{array}$ & ${ }^{0} \quad 5 \quad 10$ \\
\hline
\end{tabular}

FIGURE 7: Forest plot showing the association between serum albumin levels and severity of disease in COVID-19 patients 


\section{Cureus}

SD, standard deviation; IV, inverse variance; Cl, confidence interval; COVID-19, coronavirus disease 2019

The serum levels of all biomarkers for severe and non-severe COVID-19 patients in each study are given in Table 3. The criterion used to diagnose COVID-19 in each study is given in the Appendices section.

\begin{tabular}{|c|c|c|c|c|c|c|c|c|c|c|}
\hline \multirow{2}{*}{$\begin{array}{l}\text { Author } \\
\text { Name }\end{array}$} & \multicolumn{2}{|c|}{ No. of COVID-19 patients } & \multicolumn{2}{|c|}{ Mean serum ALT levels } & \multicolumn{2}{|c|}{ Mean serum AST levels } & \multicolumn{2}{|c|}{ Mean serum total bilirubin levels } & \multicolumn{2}{|c|}{ Mean serum albumin levels } \\
\hline & $\begin{array}{l}\text { Severe } \\
\text { CovID-19 }\end{array}$ & $\begin{array}{l}\text { Non-severe } \\
\text { Covid-19 }\end{array}$ & $\begin{array}{l}\text { Severe } \\
\text { CovID-19 }\end{array}$ & $\begin{array}{l}\text { Non-severe } \\
\text { Covid-19 }\end{array}$ & $\begin{array}{l}\text { Severe } \\
\text { CovID-19 }\end{array}$ & $\begin{array}{l}\text { Non-severe } \\
\text { CoviD-19 }\end{array}$ & $\begin{array}{l}\text { Severe } \\
\text { CovID-19 }\end{array}$ & $\begin{array}{l}\text { Non-severe } \\
\text { CoviD-19 }\end{array}$ & $\begin{array}{l}\text { Severe } \\
\text { CoviD-19 }\end{array}$ & $\begin{array}{l}\text { Non-severe } \\
\text { COVID-19 }\end{array}$ \\
\hline Cai et al. [6] & 58 & 240 & $26.85 \pm 16.32$ & $20 \pm 12.11$ & $36 \pm 15.9$ & $26 \pm 10.83$ & $11.25 \pm 7.76$ & $10.9 \pm 1.18$ & - & - \\
\hline Cai et al. [7] & 85 & 233 & $67 \pm 42.0$ & $41 \pm 33.47$ & $58 \pm 41.08$ & $34 \pm 14.41$ & $22 \pm 7.88$ & $19 \pm 10.38$ & - & - \\
\hline $\begin{array}{l}\text { Chen et } \\
\text { al. [8] }\end{array}$ & 11 & 10 & $42 \pm 12.96$ & $16 \pm 6.29$ & $47 \pm 34.44$ & $24 \pm 3.70$ & $8.80 \pm 1.92$ & $7.80 \pm 2.29$ & $29.60 \pm 3.25$ & $37.20 \pm 2.22$ \\
\hline $\begin{array}{l}\text { Chen et } \\
\text { al. [4] }\end{array}$ & 113 & 161 & $28.00 \pm 21.48$ & $20.00 \pm 12.74$ & $45.00 \pm 26.66$ & $25.00 \pm 9.85$ & $12.60 \pm 4.4$ & $8.40 \pm 4.00$ & $30.10 \pm 3.77$ & $36.30 \pm 4.29$ \\
\hline $\begin{array}{l}\text { Deng et } \\
\text { al. [9] }\end{array}$ & 109 & 116 & $22.00 \pm 14.07$ & $18.70 \pm 13.2$ & $34.00 \pm 14.81$ & $22.00 \pm 10.44$ & - & - & - & - \\
\hline Fu et al. [10] & 88 & 211 & $23.0 \pm 20.39$ & $22.0 \pm 15.99$ & $29.0 \pm 24.5$ & $26.0 \pm 11.94$ & $10.9 \pm 6.47$ & $10.4 \pm 5.75$ & $37.3 \pm 5.0$ & $40.5 \pm 5.75$ \\
\hline al. [11] & 15 & 28 & $27.00 \pm 14.81$ & $24.50 \pm 16.29$ & $27.80 \pm 11.42$ & $33.21 \pm 18.24$ & - & - & - & - \\
\hline $\begin{array}{l}\text { Huang et } \\
\text { al. [1] }\end{array}$ & 13 & 28 & $49.00 \pm 63.7$ & $27.00 \pm 15.18$ & $44.00 \pm 29.62$ & $34.00 \pm 12.22$ & $14.00 \pm 15.55$ & $10.80 \pm 2.14$ & - & - \\
\hline $\begin{array}{l}\text { Jin et } \\
\text { al. [12] }\end{array}$ & 74 & 577 & $25.00 \pm 16.82$ & $21.50 \pm 13.18$ & $29.35 \pm 13.14$ & $24.40 \pm 9.62$ & $10.00 \pm 4.92$ & $9.60 \pm 4.51$ & $40.13 \pm 4.92$ & $41.50 \pm 3.8$ \\
\hline $\begin{array}{l}\text { Lei et } \\
\text { al. [13] }\end{array}$ & 1186 & 4585 & $26.0 \pm 22.47$ & $23.0 \pm 18.31$ & $31.0 \pm 20.01$ & $22.0 \pm 11.05$ & $10.6 \pm 5.62$ & $10.3 \pm 4.84$ & - & - \\
\hline $\begin{array}{l}\text { Liu et } \\
\text { al. [14] }\end{array}$ & 11 & 67 & $17.40 \pm 22.22$ & $18.50 \pm 11.25$ & $21.60 \pm 24.88$ & $20.00 \pm 12.59$ & - & - & $36.62 \pm 6.6$ & $41.27 \pm 4.55$ \\
\hline $\begin{array}{l}\text { Mo et } \\
\text { al. [15] }\end{array}$ & 85 & 70 & $28.00 \pm 18.51$ & $20.00 \pm 13.33$ & $37.00 \pm 29.62$ & $32.00 \pm 11.11$ & - & - & $36.00 \pm 5.92$ & $39.00 \pm 4.44$ \\
\hline $\begin{array}{l}\text { Pan et } \\
\text { al. [16] }\end{array}$ & 103 & 101 & $42.24 \pm 43.83$ & $29.53 \pm 23.58$ & $35.12 \pm 26.58$ & $27.48 \pm 23.98$ & $13.83 \pm 12.03$ & $13.46 \pm 8.11$ & $36.16 \pm 6.49$ & $35.84 \pm 5.63$ \\
\hline $\begin{array}{l}\text { Qian et } \\
\text { al. [18] }\end{array}$ & 9 & 82 & $19.90 \pm 8.88$ & $18.00 \pm 11.85$ & $27.00 \pm 2.4$ & $21.00 \pm 8.88$ & - & - & $38.55 \pm 2.16$ & $40.20 \pm 3.25$ \\
\hline $\begin{array}{l}\text { Qu et } \\
\text { al. [19] }\end{array}$ & 3 & 27 & $36.00 \pm 19.52$ & $33.59 \pm 24.54$ & $45.33 \pm 12.9$ & $43.56 \pm 21.03$ & - & - & - & - \\
\hline $\begin{array}{l}\text { Ruan et } \\
\text { al. [20] }\end{array}$ & 68 & 82 & - & - & - & - & $18.10 \pm 10.7$ & $12.80 \pm 6.8$ & $28.80 \pm 3.8$ & $32.70 \pm 3.8$ \\
\hline $\begin{array}{l}\text { Wan et } \\
\text { al. [21] }\end{array}$ & 40 & 95 & $26.60 \pm 13.92$ & $21.70 \pm 16.37$ & $33.60 \pm 13.70$ & $22.40 \pm 10.07$ & $9.80 \pm 5.77$ & $8.60 \pm 6.22$ & $36.00 \pm 4.0$ & $49.90 \pm 4.59$ \\
\hline $\begin{array}{l}\text { Wang et } \\
\text { al. [22] }\end{array}$ & 36 & 102 & $35.00 \pm 28.14$ & $23.00 \pm 15.55$ & $2.00 \pm 29.62$ & $29.00 \pm 12.59$ & $11.50 \pm 6.66$ & $9.30 \pm 3.40$ & - & - \\
\hline $\begin{array}{l}\text { Wang et } \\
\text { al. [23] }\end{array}$ & 14 & 55 & $31.50 \pm 21.48$ & $24.00 \pm 17.77$ & $40.50 \pm 28.14$ & $26.00 \pm 13.33$ & - & - & - & - \\
\hline $\begin{array}{l}\text { Wu et } \\
\text { al. [24] }\end{array}$ & 84 & 117 & $35.00 \pm 22.96$ & $27.00 \pm 17.40$ & $38.00 \pm 16.66$ & $30.00 \pm 10.74$ & 12.9 & $10.50=$ & \pm 4.59 & $33.70 \pm 3.96$ \\
\hline $\begin{array}{l}\text { Xie et } \\
\text { al. [25] }\end{array}$ & 28 & 51 & $36.5 \pm 41.31$ & $30.0 \pm 17.6$ & $35 \pm 22.95$ & $28 \pm 20.34$ & $12.7 \pm 5.6$ & $13.9 \pm 7.64$ & - & - \\
\hline Yang et & 32 & 20 & - & - & - & - & $19.50 \pm 11.6$ & $13.10 \pm 4.3$ & - & - \\
\hline
\end{tabular}




\section{Cureus}

\begin{tabular}{|c|c|c|c|c|c|c|c|c|c|c|}
\hline al. [26] & & & & & & & & & & \\
\hline $\begin{array}{l}\text { Zhang et } \\
\text { al. [27] }\end{array}$ & 573 & 72 & $29.37 \pm 25.71$ & $25.53 \pm 19.96$ & $30.08 \pm 20.37$ & $25.67 \pm 15.52$ & $11.26 \pm 8.04$ & $9.11 \pm 4.86$ & $41.02 \pm 4.47$ & $42.53 \pm 4.7$ \\
\hline $\begin{array}{l}\text { Zhou et } \\
\text { al. [28] }\end{array}$ & 8 & 26 & $49.00 \pm 34.07$ & $34.00 \pm 29.62$ & $44.00 \pm 16.29$ & $32.00 \pm 14.81$ & - & - & - & - \\
\hline $\begin{array}{l}\text { Zhou et } \\
\text { al. [29] }\end{array}$ & 54 & 137 & $40.00 \pm 20.0$ & $27.00 \pm 18.51$ & - & - & - & - & $29.10 \pm 3.55$ & $33.60 \pm 4.29$ \\
\hline
\end{tabular}

TABLE 3: Serum levels of ALT, AST, total bilirubin, and albumin among severe and non-severe COVID-19 patients in the included studies

COVID-19, coronavirus disease 2019; ALT, alanine transaminase; AST, aspartate transaminase

\section{Discussion}

Our meta-analysis has three salient findings. Firstly, almost 16\% of the COVID-19 positive patients had a substantial elevation in enzymes ( $>3$ times the ULN). Secondly, the prevalence of current liver injury in hospitalized COVID-19 patients was significantly higher than the prevalence of patients with known preCOVID CLD. Thirdly, the extent of liver damage was associated with the severity of COVID-19.

Although our study demonstrates a clear association between COVID-19 and liver injury, mechanisms for liver injury remain unclear. Direct injury by SARS-CoV-2 has been proposed as a likely mechanism [3]. The expression of ACE2 protein (entry receptor for SARS-CoV-2) on bile duct cells supports the possibility of virus-mediated liver damage [3]. However, there is a lower frequency of receptors found in liver cells than bile duct cells, and trends show an elevation in aminotransferases rather than alkaline phosphate and gamma-glutamyl transferase [3,6,7,17]. Thus, alternate mechanisms must be considered as well.

Apart from direct viral-mediated injury, drug-induced liver injury must also be given consideration. Acetaminophen, a drug commonly used by COVID-19 patients, is known to cause hepatic injury at doses $>7.5$ to $10 \mathrm{~g}$ in adults [30]. In addition, the simultaneous use of multiple antiviral therapies and antibiotics in these patients can be hepatotoxic [17,21-23]. Aggressive treatment in patients with more severe disease may explain the association of liver injury with disease severity seen in our study.

Systemic effects of COVID-19 could be another possible explanation for the liver injury. It is proven that SARS-Cov-2 infects the lung causing hypoxia and, in severe cases, acute respiratory distress syndrome, sepsis, and multi-organ failure $[1,4,9,17]$. It can be imagined that sepsis in COVID-19 leads to hypoxic injury and ischemia of the liver, causing elevated liver biochemistries, which further explains why serum ALT, AST, and total bilirubin levels are higher in severe/ICU COVID-19 patients than non-severe patients, as demonstrated in our study $[6,7,17]$.

To the best of our knowledge, this is the first meta-analysis to report the prevalence of liver injury in COVID-19 patients while keeping the definition of liver injury standard and accounting for baseline CLD history. Thus, these results should be generalizable across cohorts of hospitalized COVID-19 patients. Our results should stimulate further research interest in the area in order to uncover mechanisms contributing to liver injury.

\section{Limitations}

This study has certain important limitations. Firstly, the quality of data-collection methods and data reported in individual studies cannot be ascertained. Secondly, patients can sometimes be unaware of underlying CLD (e.g., in non-alcoholic fatty liver disease), and these patients may be misclassified as having no CLD. This could have led to an underestimation of baseline CLD prevalence. Third, as shown, estimates from our study had significantly high heterogeneity. This is likely due to the inclusion of primarily small studies with varied prevalence. Lastly, all included studies were of observational nature, and there is a need for randomized trials on this aspect of COVID-19. Although meta-analysis can increase the power and provide better estimates, the results are intended to offer early insight and should not be considered a replacement for large-scale observational studies that are being awaited.

\section{Conclusions}

This systematic review and meta-analysis shows that COVID-19 is associated with an increased incidence of liver injury. Furthermore, the extent of derangement in serum liver function markers is associated with the severity of COVID-19. Future studies should adopt a pre-defined criterion for reporting liver injury and exclude those patients from the analyses that had any form of baseline derangement in liver enzymes and 


\section{Cureus}

biomarkers at hospital admission. A standard protocol should be formed for COVID-19 patients to identify them as "with liver injury" or "without liver injury", as this will lead to uniform reporting and low bias in studies.

\section{Appendices}

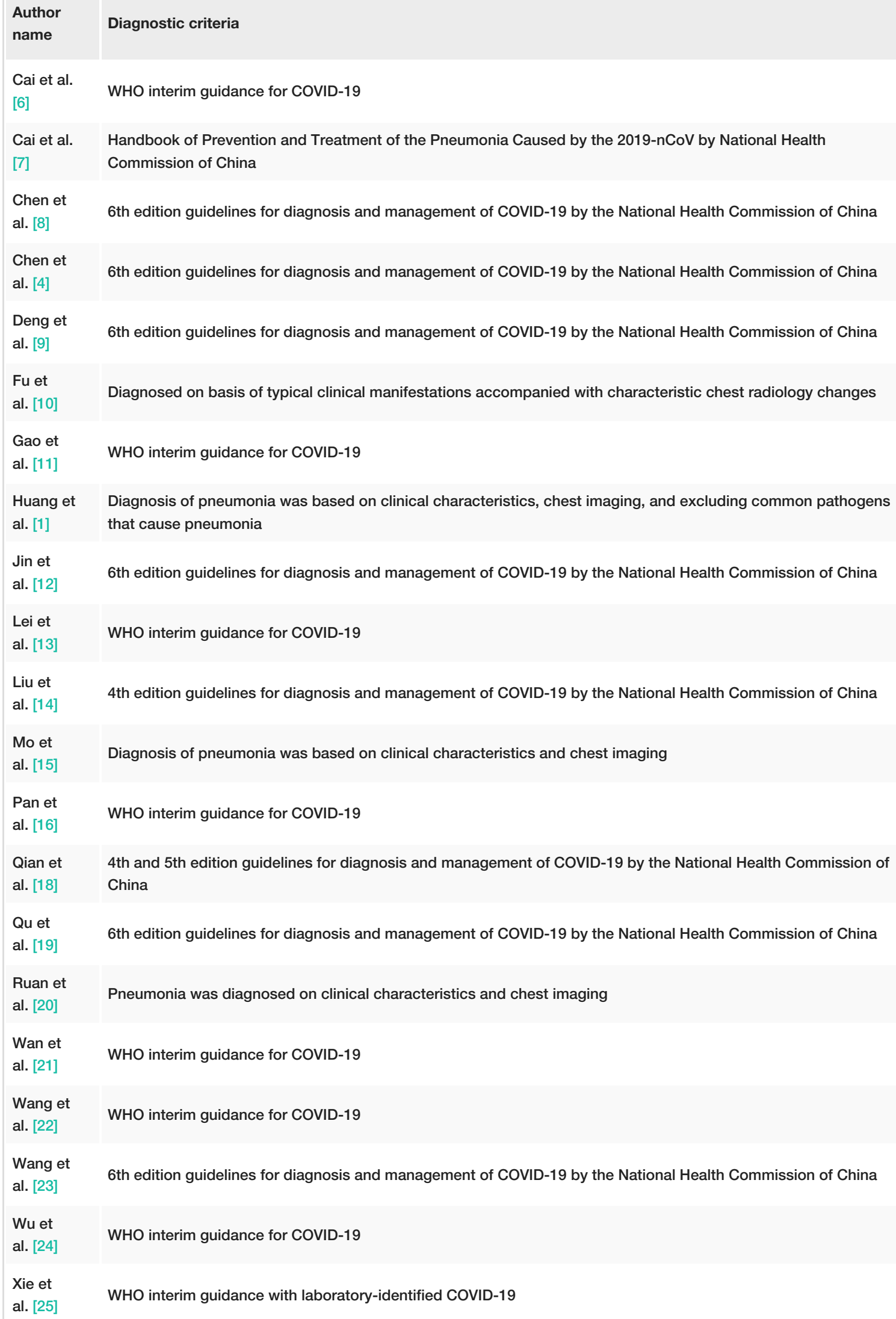




\section{Cureus}

Yang et

al. [26]

WHO interim guidance for COVID-19

Zhang et WHO interim guidance for COVID-19 and 6th edition guidelines for diagnosis and management of COVID-19 by the al. [27] National Health Commission of China

Zhou et al. [28] 4th edition guidelines for diagnosis and management of COVID-19 by the National Health Commission of China

Zhou et WHO interim guidance for COVID-19 and 6th edition guidelines for diagnosis and management of COVID-19 by the al. [29] National Health Commission of China

TABLE 4: Diagnostic criteria used for the assessment of severity of COVID-19 among patients in the included studies

WHO, World Health Organization; COVID-19, coronavirus disease 2019; 2019-nCoV, 2019 novel coronavirus

\section{Additional Information \\ Disclosures}

Human subjects: All authors have confirmed that this study did not involve human participants or tissue. Animal subjects: All authors have confirmed that this study did not involve animal subjects or tissue. Conflicts of interest: In compliance with the ICMJE uniform disclosure form, all authors declare the following: Payment/services info: All authors have declared that no financial support was received from any organization for the submitted work. Financial relationships: All authors have declared that they have no financial relationships at present or within the previous three years with any organizations that might have an interest in the submitted work. Other relationships: All authors have declared that there are no other relationships or activities that could appear to have influenced the submitted work.

\section{References}

1. Huang C, Wang Y, Li X, et al.: Clinical features of patients infected with 2019 novel coronavirus in Wuhan, China. Lancet. 2020, 395:497-506. 10.1016/S0140-6736(20)30183-5

2. Bloom PP, Meyerowitz EA, Reinus Z, et al.: Liver biochemistries in hospitalized patients with COVID-19 [Published online ahead of print]. Hepatology. 2020, 10.1002/hep.31326

3. Chai X, Hu L, Zhang Y, et al.: Specific ACE2 expression in cholangiocytes may cause liver [Published online ahead of print]. BioRxiv. 2020, 10.1101/2020.02.03.931766

4. Chen T, Wu D, Chen H, et al.: Clinical characteristics of 113 deceased patients with coronavirus disease 2019: retrospective study. BMJ. 2020, 368:1295. 10.1136/bmj.m1295

5. Hutton B, Salanti G, Caldwell DM, et al.: The PRISMA extension statement for reporting of systematic reviews incorporating network meta-analyses of health care interventions: checklist and explanations. Ann Intern Med. 2015, 162:777-784. 10.7326/M14-2385

6. Cai Q, Huang D, Ou P, et al.: COVID-19 in a designated infectious diseases hospital outside Hubei province, China [Published online ahead of print]. Allergy. 2020, 10.1111/all.14309

7. Cai Q, Huang D, Yu H, et al.: COVID-19: abnormal liver function tests [Published online ahead of print] . J Hepatol. 2020, 10.1016/j.jhep.2020.04.006

8. Chen G, Wu D, Guo W, et al.: Clinical and immunological features of severe and moderate coronavirus disease 2019. J Clin Invest. 2020, 130:2620-2629. 10.1172/JCI137244

9. Deng Y, Liu W, Liu K, et al.: Clinical characteristics of fatal and recovered cases of coronavirus disease 2019 in Wuhan, China: a retrospective study. Chin Med J. 2020, 133:1261-1267. 10.1097/CM9.0000000000000824

10. Fu L, Fei J, Xu S, et al.: Acute liver injury and its association with death risk of patients with COVID- 19: a hospital-based prospective case-cohort study [Published online ahead of print]. medRxiv. 2020, 10.1101/2020.04.02.20050997

11. Gao Y, Li T, Han M, et al.: Diagnostic utility of clinical laboratory data determinations for patients with the severe COVID-19. J Med Virol. 2020, 92:791-796. 10.1002/jmv.25770

12. Jin X, Lian JS, Hu JH, et al.: Epidemiological, clinical and virological characteristics of 74 cases of coronavirus-infected disease 2019 (COVID-19) with gastrointestinal symptoms. Gut. 2020, 69:1002-1009. 10.1136/gutjnl-2020-320926

13. Lei F, Liu YM, Zhou F, et al.: Longitudinal association between markers of liver injury and mortality in COVID-19 in China [Published online ahead of print]. Hepatology. 2020, 10.1002/hep.31301

14. Liu W, Tao ZW, Wang L, et al.: Analysis of factors associated with disease outcomes in hospitalized patients with 2019 novel coronavirus disease. Chin Med J. 2020, 133:1032-1038. 10.1097/CM9.0000000000000775

15. Mo P, Xing Y, Xiao Y, et al.: Clinical characteristics of refractory COVID-19 pneumonia in Wuhan, China [Published online ahead of print]. Clin Infect Dis. 2020, ciaa270. 10.1093/cid/ciaa270

16. Pan L, Mu M, Yang P, et al.: Clinical characteristics of COVID-19 patients with digestive symptoms in Hubei, China: a descriptive, cross-sectional, multicenter study. Am J Gastroenterol. 2020, 115:766-773. 10.14309/ajg.0000000000000620

17. Phipps MM, Barraza LH, LaSota ED, et al.: Acute liver injury in COVID- 19: prevalence and association with clinical outcomes in a large US cohort [Published online ahead of print]. Hepatology. 2020, 


\section{Cureus}

10.1002/hep.31404

18. Qian GQ, Yang NB, Ding F, et al.: Epidemiologic and clinical characteristics of 91 hospitalized patients with COVID-19 in Zhejiang, China: a retrospective, multi-centre case series [Published online ahead of print]. QJM. 2020, 113:474-481. 10.1093/qjmed/hcaa089

19. Qu R, Ling Y, Zhang YH, et al.: Platelet-to-lymphocyte ratio is associated with prognosis in patients with coronavirus disease-19 [Published online ahead of print]. J Med Virol. 2020, 10.1002/jmv.25767

20. Ruan Q, Yang K, Wang W, Jiang L, Song J: Clinical predictors of mortality due to COVID-19 based on an analysis of data of 150 patients from Wuhan, China. Intensive Care Med. 2020, 46:846-848. 10.1007/s00134020-05991-X

21. Wan S, Xiang Y, Fang W, et al.: Clinical features and treatment of COVID-19 patients in northeast Chongqing. J Med Virol. 2020, 92:797-806. 10.1002/jmv.25783

22. Wang D, Hu B, Hu C, et al.: Clinical characteristics of 138 hospitalized patients with 2019 novel coronavirus-infected pneumonia in Wuhan, China. JAMA. 2020, 323:1061-1069. 10.1001/jama.2020.1585

23. Wang Z, Yang B, Li Q, Wen L, Zhang R: Clinical features of 69 cases with coronavirus disease 2019 in Wuhan, China [Published online ahead of print]. Clin Infect Dis. 2020, 10.1093/cid/ciaa272

24. Wu C, Chen X, Cai Y, et al.: Risk factors associated with acute respiratory distress syndrome and death in patients with coronavirus disease 2019 pneumonia in Wuhan, China [Published online ahead of print]. JAMA Intern Med. 2020, e200994. 10.1001/jamainternmed.2020.0994

25. Xie H, Zhao J, Lian N, Lin S, Xie Q, Zhuo H: Clinical characteristics of non-ICU hospitalized patients with coronavirus disease 2019 and liver injury: a retrospective study. Liver Int. 2020, 40:1321-1326. 10.1111/liv.14449

26. Yang X, Yu Y, Xu J, et al.: Clinical course and outcomes of critically ill patients with SARS-CoV-2 pneumonia in Wuhan, China: a single-centered, retrospective, observational study. Lancet Respir Med. 2020, 8:475-481. 10.1016/S2213-2600(20)30079-5

27. Zhang X, Cai H, Hu J, et al.: Epidemiological, clinical characteristics of cases of SARS-CoV-2 infection with abnormal imaging findings. Int J Infect Dis. 2020, 94:81-87. 10.1016/j.ijid.2020.03.040

28. Zhou B, She J, Wang Y, Ma X: The clinical characteristics of myocardial injury in severe and very severe patients with 2019 novel coronavirus disease. J Infect. 2020, 81:168-170. 10.1016/j.jinf.2020.03.021

29. Zhou F, Yu T, Du R, et al.: Clinical course and risk factors for mortality of adult inpatients with COVID-19 in Wuhan, China: a retrospective cohort study. Lancet. 2020, 395:1054-1062. 10.1016/S01406736(20)30566-3

30. Hodgman MJ, Garrard AR: A review of acetaminophen poisoning . Crit Care Clin. 2012, 28:499-516. 10.1016/j.ccc.2012.07.006 